\title{
Effect of iron overload on impaired fertility in male patients with transfusion-dependent beta-thalassemia
}

\author{
Mei-Jou Chen ${ }^{1}$, Steven Shinn-Forng Peng ${ }^{2}$, Meng-Yao Lu ${ }^{3}$, Yung-Li Yang ${ }^{4}$, Shiann-Tarng Jou ${ }^{3}$, Hsiu-Hao Chang ${ }^{3}$, \\ Shee-Uan Chen ${ }^{1}$, Dong-Tsamn Lin ${ }^{4}$ and Kai-Hsin Lin $^{3}$
}

BACKGROUND: To investigate the fertility of male patients with transfusion-dependent beta-thalassemia, and to use magnetic resonance imaging $(\mathrm{MRI})$ as a novel method to assess the iron overload status of testis in such patients.

METHODS: Twenty-one male patients with transfusiondependent beta-thalassemia and five normal male controls enrolled in this study. Hormonal profiles, iron levels, MRI testicular dimension, MRI T2 values, parameters for sperm quality, and sperm DNA fragmentation (SDF) of participants were measured.

RESULTS: The MRI T2 values of the testis were significantly lower in transfusion-dependent beta-thalassemia patients than in normal controls $(P=0.027)$, and they correlated to serum ferritin levels in all enrolled subjects $\left(R^{2}=0.258\right.$, $P=0.008)$. There were significantly lower sperm concentrations $(P=0.037)$, a lower percentage of sperm with normal morphology $(P=0.001)$, and a higher percentage of SDF $(P=0.009)$ in transfusion-dependent beta-thalassemia patients without hypogonadotropic hypogonadism and with spontaneous spermatogenesis compared with normal controls. The percentage of SDF was significantly correlated with serum ferritin levels in transfusion-dependent beta-thalassemia male patients with spontaneous spermatogenesis $\left(R^{2}=0.48\right.$, $P=0.009)$.

CONCLUSION: Our study is the first demonstration of iron deposition in the testis of patients with transfusion-dependent beta-thalassemia based on imaging, and such findings might explain the high prevalence of impaired fertility in above patients with normal pituitary function.

B eta-thalassemia is a disorder of hereditary chronic anemia derived from a defect in beta-globin chain production (1). Transfusion-dependent beta-thalassemia, also known as betathalassemia major, most often results from homozygosity or compound heterozygosity of a mutant beta-thalassemia allele. Most patients with transfusion-dependent beta-thalassemia require regular red blood cell transfusions, which starts within the first year of life $(2,3)$. Repeated red blood cell transfusions lead to iron deposition in various organs and tissues, primarily including the liver, heart, and endocrine glands, thus causing tissue damage and organ dysfunction (1). Although the combination of transfusion and iron-chelating therapy has markedly extended the life expectancy of these patients, iron overload after repeated red blood cell transfusions causes long-term morbidity and mortality.

A lack of sexual maturation and loss of gonadal function occur frequently in these patients, which may reflect the high prevalence of hypogonadotropic hypogonadism $(4,5)$ and the predilection for iron deposition in the pituitary gland and hypothalamus (6). Controversy still exists with respect to the causes of gonadal dysfunction due to primary iron deposits in the gonads or secondary to a hypogonadotropic state in patients with beta-thalassemia major (7-9). Whereas pregnancy has been reported in women with transfusiondependent beta-thalassemia (10), paternity is less common in men with transfusion-dependent beta-thalassemia and has been addressed infrequently (11). Transfusions and advances in iron-chelating therapy have significantly improved the long-term survival and quality of life for patients with transfusion-dependent beta-thalassemia $(12,13)$; therefore, the preservation of reproductive function and evaluation of male patients with transfusion-dependent beta-thalassemia have become an important issue $(14,15)$.

The role of iron in the formation of reactive oxygen species (ROS), including free radicals $(16,17)$ in biologic systems that result in human diseases, is well known (18). The harmful effects of ROS on the sperm membrane, structural components, and nucleus have also been reported (19). Previous studies have demonstrated oligoasthenospermia $(20,21)$ and sperm DNA damage $(17,22)$ in male patients with transfusion-dependent beta-thalassemia, and reported a decline

\footnotetext{
'Department of Obstetrics and Gynecology and Livia Shangyu Wan Scholar, National Taiwan University Hospital, National Taiwan University, College of Medicine, Taipei, Taiwan; ${ }^{2}$ Department of Medical Imaging, National Taiwan University Hospital, National Taiwan University, College of Medicine, Taipei, Taiwan; ${ }^{3}$ Department of Pediatrics, National Taiwan University Hospital, National Taiwan University, College of Medicine, Taipei, Taiwan; ${ }^{4}$ Department of Laboratory Medicine, National Taiwan University Hospital, National Taiwan University, College of Medicine, Taipei, Taiwan. Correspondence: Hsiu-Hao Chang (changhh2001@ntu.edu.tw)

The first two authors contributed equally to this work

H.-H.C. and M.-J.C. designed the study, recruited patients, analyzed and interpreted data, and drafted the manuscript. S.S.-F.P. collected and analyzed MRI data and drafted the manuscript. All the authors participated in the interpretation of data and manuscript drafting, editing, and critical discussion.

Received 12 July 2017; accepted 29 October 2017; advance online publication 13 December 2017. doi:10.1038/pr.2017.296
} 


\section{Articles | Chen et al.}

in testicular function in such patients; however, whether or not gonadal dysfunction in transfusion-dependent beta-thalassemia due to direct iron overload on the testis has not been determined.

The conventional tool to evaluate the iron overload status is to measure the serum ferritin level in patients with transfusion-dependent beta-thalassemia; however, the serum ferritin level cannot reflect the iron status in specific organs. Magnetic resonance imaging (MRI) is now widely used in the assessment of organ-specific iron overload, and helps to improve patient compliance with iron chelation therapy (23), but has not yet been used to evaluate the iron burden in male gonads. In this study, we determined the fertility of male patients with transfusion-dependent beta-thalassemia in terms of semen quality and integrity of sperm DNA, and used MRI as a novel method to assess the iron content status of testis in such patients.

\section{METHODS}

\section{Study Design and Population}

Male patients with transfusion-dependent beta-thalassemia, $>20$ years of age, were enrolled non-selectively in the study. All patients received blood transfusions at regular intervals (every 4-5 weeks) to maintain a hemoglobin level of at least $9.5 \mathrm{gm} / \mathrm{dl}$ before each transfusion. Desferoxamine, deferiprone, or deferasirox were used as iron-chelating therapy depending on the serum ferritin level and patient tolerance. No participants were on steroid treatment or medications known to cause hyperprolactinemia or other known endocrinopathies. Patients with a history of genital surgery, epididymo-orchitis, varicocele, drug abuse, tobacco use, venereal disease, or concomitant medical problems known to be associated with decreased fertility were excluded from this study. Controls consisted of healthy, age-matched male volunteers without a history of problems with puberty or fertility. This study was approved by the Institutional Review Board of the National Taiwan University Hospital. Every participant provided written informed consent.

\section{Patient Assessment}

The clinical data, including blood transfusion, average serum ferritin levels for the previous 12 months, most recent cardiac T2* value within previous 12 months, and iron overload-related morbidities (hepatitis $\mathrm{C}$ virus infection, diabetes, and hypogonadotrophic hypogonadism) of patients with transfusion-dependent beta-thalassemia at the time of blood sampling, were obtained by reviewing the medical records. We defined the clinical diagnosis of diabetes mellitus as a fasting plasma glucose $\geqq 126 \mathrm{mg} / \mathrm{dl}$. Hypogonadotropic hypogonadism was defined as luteinizing hormone (LH) and folliclestimulating hormone $(\mathrm{FSH})$ levels $<2 \mathrm{IU} / \mathrm{l}$, a testosterone concentration $<3 \mathrm{ng} / \mathrm{ml}$, no spontaneous spermatogenesis, and an abnormal LH response to the LH-releasing hormone test.

Blood samples were obtained from all participants for laboratory investigations, which included serum levels of LH, FSH, testosterone, prolactin, estradiol, inhibin B, and ferritin. Semen samples were also obtained from all participants. Each participant collected one semen sample into a sterile container after at least $48 \mathrm{~h}$ of abstinence from ejaculation. Most samples were provided on site or were delivered to the laboratory within $60 \mathrm{~min}$ of collection. Semen samples were then analyzed in accordance with the World Health Organization (WHO) guidelines $(24,25)$. Integrity of sperm DNA was assessed by the sperm chromatin dispersion test using the Halosperm kit (INDAS Laboratories, Madrid, Spain) to analyze sperm DNA fragmentation (SDF) (26). Briefly, intact spermatozoa were immersed in an agarose matrix on a slide that was pretreated with an acid solution to denature DNA in those sperm cells with fragmented DNA. Then, the slide was treated with lysis buffer to remove nuclear membranes and proteins. Removal of nuclear proteins resulted in nucleoids with a central core and a peripheral halo of dispersed DNA loops. In the absence of massive DNA breakage, it produced nucleoids with large halos of spreading DNA loops, emerging from a central core. However, the nucleoids from spermatozoa with fragmented DNA either do not show a dispersion halo or the halo is minimal. A minimum of 500 spermatozoa per sample was scored for percentage of SDF under the $\times 100$ objective of the microscope (27). The timing of blood and semen sample collection was before red blood cell transfusion for patients with transfusion-dependent betathalassemia.

The MRI T2 values of the testis were assessed with a 1.5-Tesla MRI scanner (GE Signa HDx, 1.5 T MRI; GE Healthcare, Milwaukee, WI) using breath-hold, multiecho, multiplanar spin-echo (MEMPSE) pulse sequence (28) in all participants. Details of the MRI parameters have been previously described (28). Axial MEMPSE images were acquired using the following parameters after instructing the patients to hold their breath: time to repeat $(\mathrm{TR})=300 \mathrm{~ms}$; time to echo $(\mathrm{TE})=3,5,8,12,18$, and $30 \mathrm{~ms}$; slice thickness/spacing between slices $=10 \mathrm{~mm} / 5 \mathrm{~mm}$; bandwidth $=62.5 \mathrm{~Hz}$; field of view $=48 \mathrm{~cm}$; matrix $=64 \times 64$, slice number $=8$; and scan time $=16 \mathrm{~s} \times 7$. A $1,000 \mathrm{ml}$ bag of normal saline solution was imaged with each patient to provide an external long T2 reference for correcting instrumental gain drift and signal intensity variations caused by bandwidth changes, as previously described (28). T2 measurements were recorded of the slice containing the mid-portion of both testes. An experienced radiologist (experience of T2 measurement for $>8$ years) who was blinded to the results of patient gonadal function and ferritin levels performed the T2 measurement by using the region of interest method. The region of interest was defined by drawing an area within the central portion while avoiding the vascular structures, as shown in Figure 1. A single exponential curve-fitting method was used, as expressed in Equation (1):

$$
S=S_{0} \mathrm{e}^{-T E / \mathrm{T} 2}
$$

where $S$ represents the observed signal intensity, $S_{0}$ is the signal intensity at $\mathrm{TE}=0, \mathrm{~T} 2$ is the transverse relaxation time, and $\mathrm{TE}$ represents the echo times. The $\mathrm{T} 2$ value of each participant was calculated by the average of T2 measurements for three slices near the mid-portion of both testes. The testicular size (dimension) was measured and defined as the longest diameter of the testis by MRI.

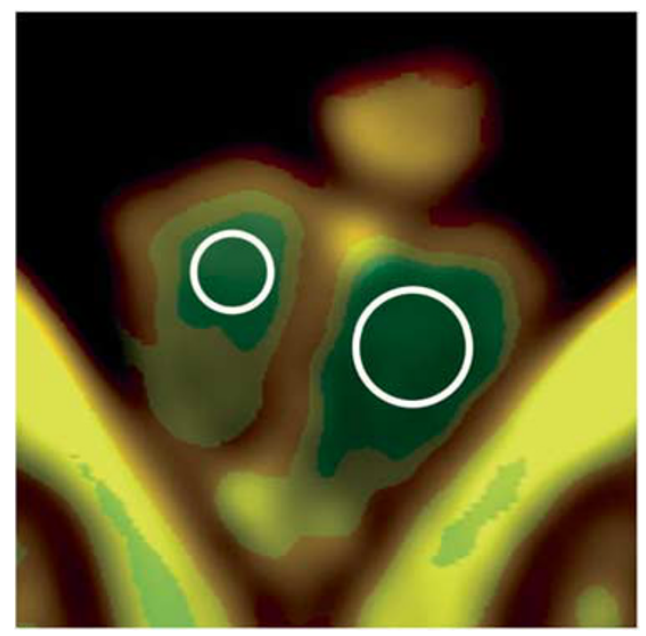

Figure 1. MEMPSE T2 map performed in the axial plane of a 32-yearold male with transfusion-dependent beta-thalassemia. Region of interest is drawn at the central portion of the mid-testis. MEMPSE, multiecho, multiplanar spin-echo. 


\section{Statistical Analysis}

The numeric variables are presented as the untransformed median and range. The categorical variables are presented as the number with the percentage in parentheses. The Shapiro-Wilk W test was used to identify whether or not all of the variables are normally distributed. Log transformation was performed on variables with a significant deviation from a normal distribution before further analysis. Non-parametric testing was applied for comparisons of age between the patient and control groups. Student's $t$-test or one-way ANOVA was applied for comparisons of parameters after log transformation between the patient and control groups. The Spearman's rank correlation and univariate linear regression analyses were performed to assess the relationship between biochemical parameters, MRI T2 values, and seminologic parameters after log transformation to correct the heterogeneity of variance. A statistically significant difference was defined as a $P<0.05$.

\section{RESULTS}

\section{Clinical Characteristics}

There were 21 male patients with transfusion-dependent betathalassemia and five normal healthy male controls enrolled in this study. Among 21 patients, 7 had hypogonadotropic hypogonadism and were under regular testosterone replacement (Table 1). Therefore, the prevalence of hypogonadotropic hypogonadism was $33.3 \%$ (7/21) among our patients. The age distributions were not different between patients and normal controls. The clinical characteristics of all enrolled subjects are listed in Table 1.

\section{Endocrine, Gonadal, and Iron Profiles}

The hormonal profiles, serum ferritin levels, MRI testicular dimension, and T2 values of our study subjects were validated to be not normally distributed by Shapiro-Wilk W test. Therefore, we compared above parameters between patients and normal control subjects by Student's $t$-test after log transformation. The LH, testosterone, prolactin, and serum ferritin levels were significantly higher in transfusiondependent beta-thalassemia male patients without hypogonadotropic hypogonadism than normal controls (Table 2). The FSH, estradiol, inhibin B levels, and the MRI testicular dimension revealed no significant difference between transfusion-dependent beta-thalassemia male patients without hypogonadotropic hypogonadism and normal controls (Table 2). The mean serum ferritin levels of all our 21 transfusion-dependent beta-thalassemia patients $(2116.7 \mathrm{ng} /$ $\mathrm{ml}$, range $541-12,932 \mathrm{ng} / \mathrm{ml}$ ) were significantly higher $(P<0.01)$ than those of normal controls $(239 \mathrm{ng} / \mathrm{ml}$, range 170-304 ng/ml). In addition, the MRI T2 values of the testis were significantly lower in transfusion-dependent betathalassemia patients than in normal controls (Figure 2a). Furthermore, the MRI T2 values of the testis were significantly, but modestly, correlated with the serum ferritin levels in all enrolled subjects $\left(R^{2}=0.258, P=0.008\right.$, Figure 2b). Among our 21 patients with transfusiondependent beta-thalassemia, the MRI T2 values of the testis were not correlated with cardiac $\mathrm{T} 2{ }^{*}$ values $\left(R^{2}=0.007\right.$, $P=0.726$, Figure 2c).

\section{Sperm Quality Assessment}

Among the 14 transfusion-dependent beta-thalassemia male patients without hypogonadotropic hypogonadism, 13 patients were noted to have spontaneous spermatogenesis and 1 patient had azoospermia. According to the $\mathrm{WHO}$ reference values for normal human semen characteristics (29), three $(23.1 \%)$ patients had low sperm concentration $\left(<15 \times 10^{6} / \mathrm{ml}\right)$, five $(38.5 \%)$ patients had low percentage of total motile sperm $(<40 \%)$, six $(46.2 \%)$ patients had low percentage of sperm with rapid motility $(<32 \%)$, and two (15.4\%) patients had low percentage of sperm with morphologically normal forms $(<4 \%)$ among the 13 transfusiondependent beta-thalassemia patients without hypogonadotropic hypogonadism and with spontaneous spermatogenesis. The above parameters for sperm quality were all in normal range for our control subjects. In addition, the parameters for sperm quality, including percentage of SDF, of our study subjects were validated to be not normally distributed by Shapiro-Wilk W test. We then compared these parameters of

Table 2. Endocrine, gonadal, and iron profiles for transfusiondependent beta-thalassemia patients without hypogonadotropic hypogonadism and normal control subjects

\begin{tabular}{|c|c|c|c|}
\hline & \multicolumn{2}{|c|}{ Mean (range) } & \multirow[t]{2}{*}{$P$ value } \\
\hline & $\begin{array}{l}\text { TDbT without } \mathrm{HH} \\
\qquad(n=14)\end{array}$ & $\begin{array}{c}\text { Normal controls } \\
\quad(n=5)\end{array}$ & \\
\hline LH (mlU/ml) & $5.8(3.9-9.5)$ & $3.1(2-4.9)$ & 0.001 \\
\hline $\mathrm{FSH}(\mathrm{mlU} / \mathrm{ml})$ & $7.1(3.5-14.9)$ & $3.9(2-6.1)$ & 0.78 \\
\hline $\begin{array}{l}\text { Testosterone } \\
(\mathrm{ng} / \mathrm{ml})\end{array}$ & $4.6(1.8-7.3)$ & $2.9(1.8-4.6)$ & 0.032 \\
\hline $\begin{array}{l}\text { Prolactin (ng/ } \\
\mathrm{ml})\end{array}$ & $10.5(5.1-17.2)$ & $5.4(4.5-7.7)$ & 0.002 \\
\hline $\begin{array}{l}\text { Estradiol (pg/ } \\
\mathrm{ml} \text { ) }\end{array}$ & $29.5(22-42.9)$ & $28.2(0-48.7)$ & 0.21 \\
\hline $\begin{array}{l}\text { Inhibin B (pg/ } \\
\text { ml) }\end{array}$ & 512.6 (172-758) & $505.8(392-676)$ & 0.908 \\
\hline Testis size $(\mathrm{cm})$ & $3.3(2.4-4)$ & $3.2(2.7-3.6)$ & 0.544 \\
\hline $\begin{array}{l}\text { Serum ferritin } \\
(\mathrm{ng} / \mathrm{ml})\end{array}$ & $1601.4(704-3,794)$ & $239(170-304)$ & $<0.001$ \\
\hline
\end{tabular}

FSH, follicle-stimulating hormone; HH, hypogonadotropic hypogonadism; LH, luteinizing hormone; TDbT, transfusion-dependent beta-thalassemia.

Table 1. Clinical characteristics of male patients with transfusion-dependent thalassemia and normal control subjects

\begin{tabular}{lcc}
\hline & Patients $(n=21)$ & Control $(n=5)$ \\
\hline Age in years (median, range) & $24.6(20.8-37.3)$ & $31.4(20.1-34.8)$ \\
Hypogonadotrophic hypogonadism & 7 & 0 \\
Hepatitis C & 2 & 0 \\
Diabetes & 5 & 0 \\
\hline
\end{tabular}




\section{Articles | chen et al.}

the 13 transfusion-dependent beta-thalassemia patients without hypogonadotropic hypogonadism and with spontaneous spermatogenesis to normal control subjects by Student's $t$-test after $\log$ transformation (Figure 3 ). There were significantly
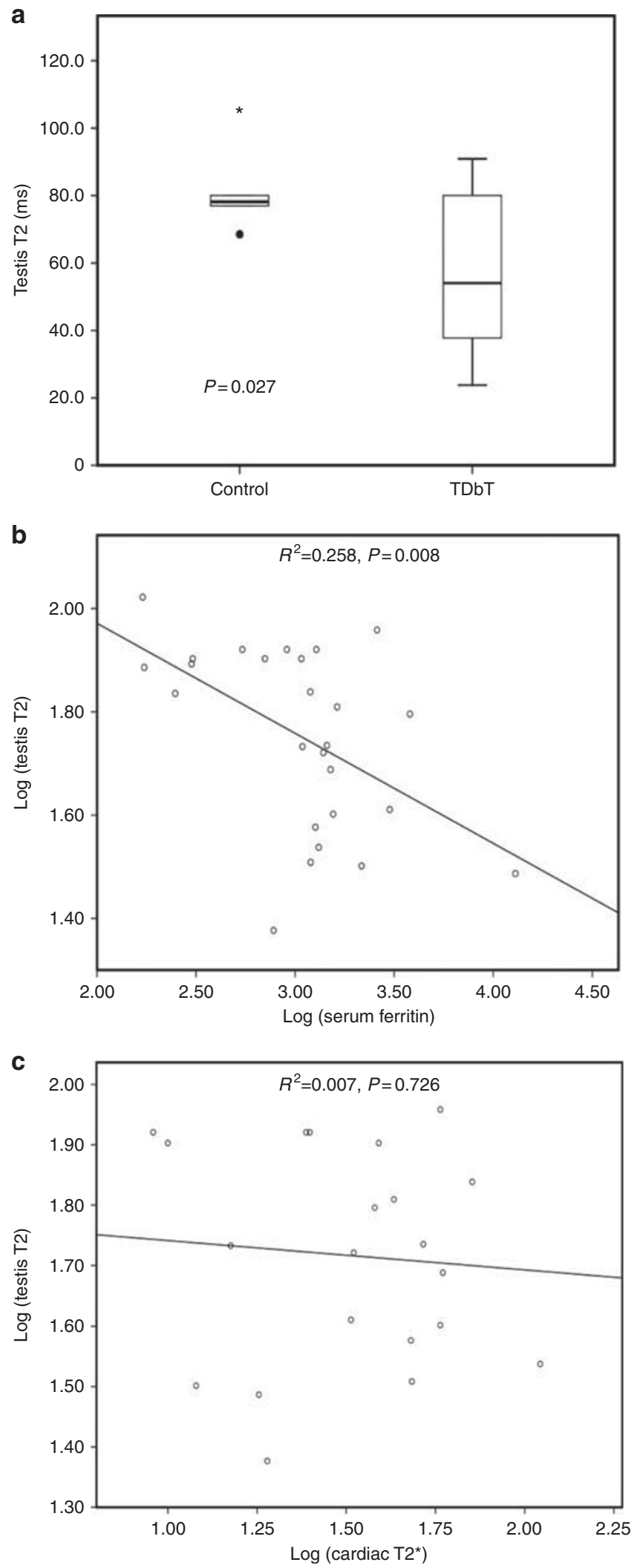

lower sperm concentrations, a lower percentage of sperm with normal morphology, and a higher percentage of SDF in transfusion-dependent beta-thalassemia patients without hypogonadotropic hypogonadism and with spontaneous spermatogenesis than in normal controls (Figure 3). There were no significant differences in the percentage of total motile sperm and the rapid motility of sperm between study and control subjects. For the MRI T2 values of testis, they were significantly lower in transfusion-dependent betathalassemia patients with spontaneous spermatogenesis than in normal controls (Figure 3).

The FSH levels were negatively correlated with the sperm concentration in transfusion-dependent beta-thalassemia patients without hypogonadotropic hypogonadism and with spontaneous spermatogenesis $\left(R^{2}=0.778, \quad P<0.01\right.$, Figure 4a). The percentage of SDF was significantly correlated with serum ferritin levels in these patients $\left(R^{2}=0.48, P=0.009\right.$, Figure $\left.4 \mathbf{b}\right)$.

\section{DISCUSSION}

Men with hypogonadotropic hypogonadism are unable to have spontaneous spermatogenesis and androgen production. Because pituitary function is vulnerable to iron deposition in iron overload syndrome, transfusion-dependent beta-thalassemia males have a high prevalence of hypogonadotropic hypogonadism due to dysfunction of the pituitary gland (30). In the current study, we found that the sperm concentration and proportion of sperms with normal morphology were significantly lower, but the SDF rate was significantly higher in transfusion-dependent beta-thalassemia male patients without hypogonadotropic hypogonadism than in normal healthy men. In addition, iron loading, as represented by elevated circulating ferritin levels, was significantly and positively correlated with the SDF proportion in transfusion-dependent beta-thalassemia males without hypogonadotropic hypogonadism. The above findings confirm that even though pituitary function might be spared from elevated iron loading after chronic blood transfusion in transfusiondependent beta-thalassemia patients undergoing ironchelating therapy and medical care, the gonadal function in males might be sensitive to and adversely affected by chronic iron loading.

Iron exhibits ferromagnetic characteristics, relaxes water hydrogen, and has been proposed to act like a magnetic resonance $(\mathrm{MR})$ contrast agent. Therefore, iron could be detectable in vivo using MR T2 or R2 quantification (31). Using the MR T2 or R2 technique is considered the current gold standard method for detection and quantification of iron

Figure 2. Distributions of iron profiles in our study subjects. (a) Distributions of testis MRI T2 value in patients with transfusiondependent beta-thalassemia (TDbT; $n=21$ ) and normal control subjects $(n=5)$. The MRI T2 values of testis were significantly lower in patient with TDbT than in controls. (b) Correlation between the MRI T2 value of testis and serum ferritin level in all enrolled subjects $(n=26)$. (c) Correlation between the MRI T2 values of testis and cardiac T2* values in patient with TDbT $(n=21)$. MRI, magnetic resonance imaging. 

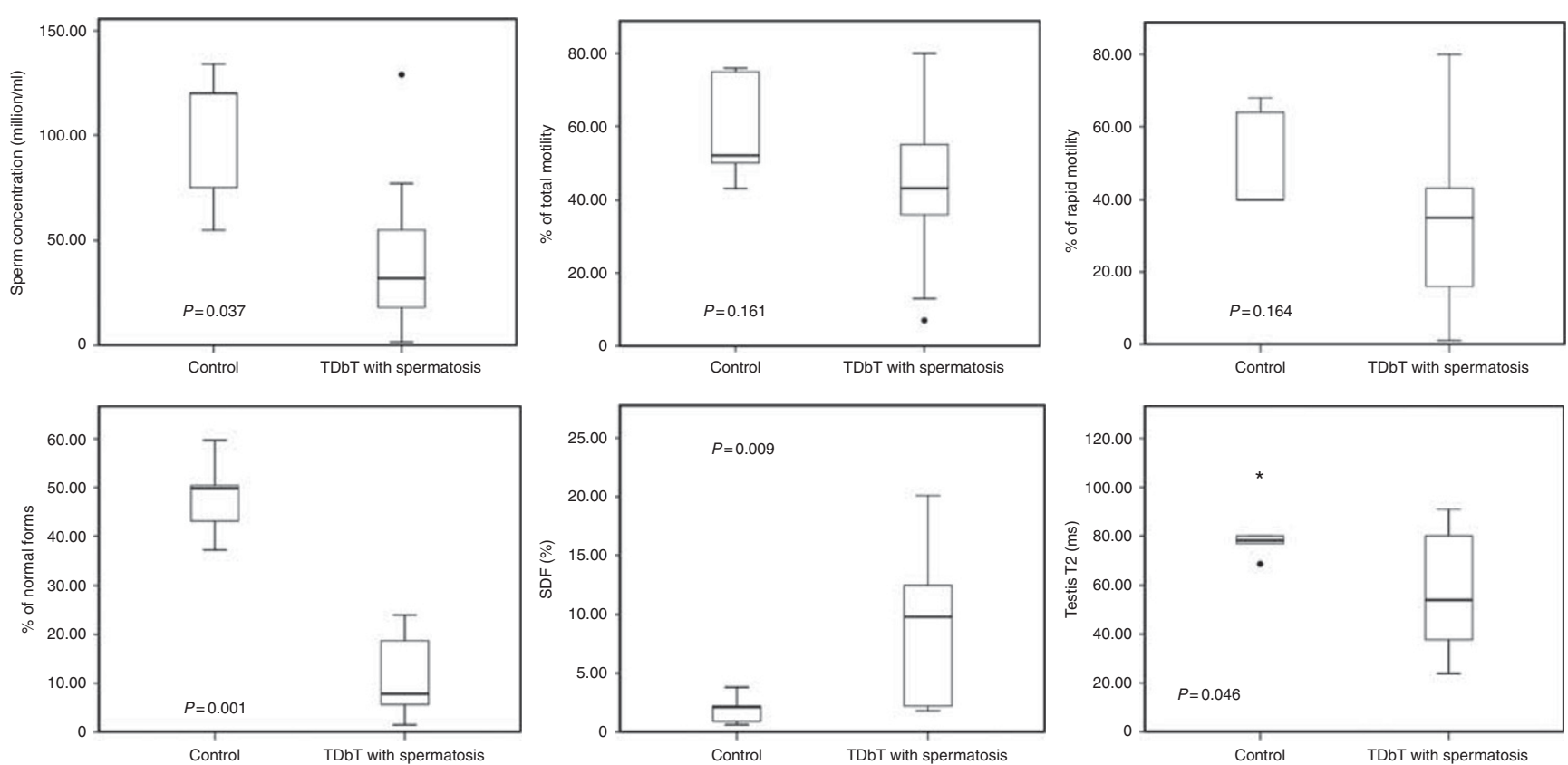

Figure 3. Semen parameters, sperm DNA fragmentation (SDF), and MRI T2 value of testis in transfusion-dependent beta-thalassemia (TDbT) patients with spontaneous spermatogenesis $(n=13)$ and in normal control subjects $(n=5)$. MRI, magnetic resonance imaging.
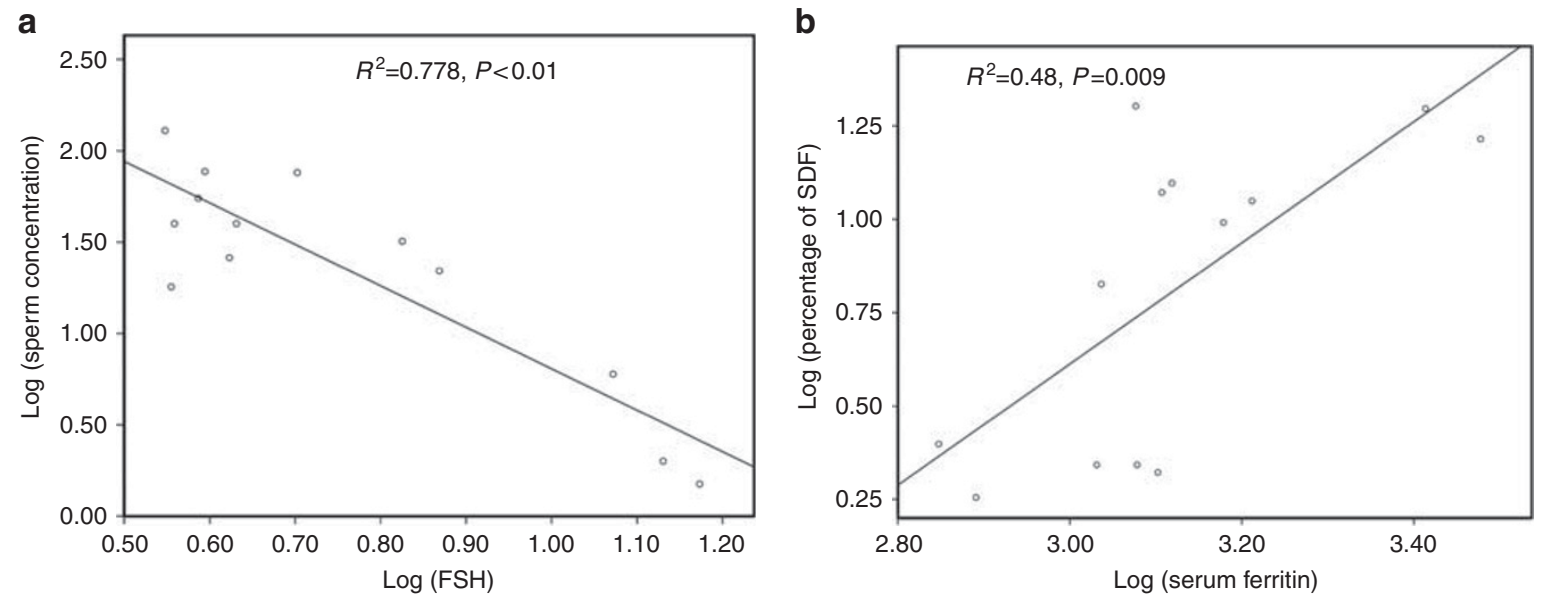

Figure 4. Distributions of endocrine, sperm quality and iron profiles in transfusion-dependent beta-thalassemia male patients without hypogonadotropic hypogonadism and with spontaneous spermatogenesis $(n=13)$. (a) Correlation between the sperm concentration and serum follicle-stimulating hormone (FSH) value in above patients. (b) Correlation between the percentage of sperm DNA fragmentation (SDF) and serum ferritin level in above patients.

deposition in myocardium, liver, and brain tissue in patients with iron overload, and has been validated by histologic evaluation (32-34). The MRI T2 and R2 techniques have also been applied to determine the severity of the iron burden causing liver and cardiac injury, and to evaluate the efficacy of iron chelator treatment in patients with thalassemia major $(23,35)$. A small series of seven male patients with transfusion-dependent thalassemia that compared the MRI R2 value of the anterior pituitary gland to sperm quality reported a relatively higher MRI R2 value of the anterior pituitary gland in male patients with concurrent high ferritin and low FSH/LH levels in three patients with azoospermia
(14). The MRI T2 technique has not yet been applied to investigate the association between iron loading and gonadal function. Our study is the first study to apply the MRI T2 technique in investigating the iron deposition of the testis.

The T2 transverse relaxation time and the inverse rate $\left(R^{2}\right.$ $(=1 / \mathrm{T} 2))$ are both conventional quantifiable MRI measures to be utilized as a surrogate to assess iron deposition. Serum ferritin has been found to have poor correlation with the MRI findings of the heart and liver in patients with iron overload disorders (36). In this study, we found a significant, although modest, correlation between ferritin levels and the MRI T2 values of the testis in all study subjects. This finding may be 


\section{Articles | Chen et al.}

because of different human tissues have different iron uptake/ clearance kinetics, which have been found in other studies $(36,37)$. In addition, like previous studies that reported a higher MRI R2 value in the vulnerable organs (heart, liver, and pituitary gland) in patients with thalassemia major $(14,23,35)$, the MRI T2 value of the testis revealed a significantly lower level in transfusion-dependent betathalassemia males compared with that in control subjects in this study. These findings suggest the MRI T2 technique is a novel and capable tool to detect and evaluate iron overload status in male gonads for patients with transfusion-dependent beta-thalassemia.

Transfusion-related iron overload in relationship to the decreased reproductive capacities has been reported both in males and females with transfusion-dependent beta-thalassemia $(14,21,22,38,39)$. Some studies $(21,38,39)$ have reported an inverse relationship between gonadal function and iron loading represented by the surrogate-circulating reproductive hormone and iron profiles as testosterone, estradiol, antiMullerian hormone, and ferritin levels. Previous studies have reported that nearly one-half of males with transfusiondependent beta-thalassemia have oligospermia/azoospermia and abnormal sperm quality that is primarily due to concurrent hypogonadotropic hypogonadism $(14,21,22)$ from iron overload. Previous studies have also reported an increased sperm DNA damage ratio, an increased proportion of abnormal sperm morphology, a lower sperm concentration, and lower motility in males with transfusion-dependent betathalassemia than age-similar control subjects. Such impairment of testicular function may be attributed to the damage of iron on the pituitary gland indirectly or on the testis directly. The detrimental effect of iron overload on the pituitary gland and testis might be due to oxidative stress through the accumulation of ROS production, generation of hydrozylation radicals, and mitochondrial function disturbance $(40,41)$ as also has been demonstrated on various tissues, including hepatocytes, pancreatic cells, endothelial cells, and thyroid gland in patients with transfusion-dependent beta-thalassemia and the general population $(1,42,43)$. In this study we demonstrated that such an inverse association between ferritin and the reported sperm function parameters still existed in transfusion-dependent beta-thalassemia male patients with normal pituitary function and spermatogenesis. Some studies showed that both Sertoli cells and sperms in testis expressed voltage-gated calcium channels on their plasma membrane $(44,45)$, which may become the portals for iron entry in iron overload condition as seen in other tissues (46). This could explain our observations that testis and its function are vulnerable to the iron overload status.

The limitations of this study included the small number of enrolled subjects, the concentrations of ROS, antioxidants, and the unavailability of iron in semen; moreover, the confounding effect of iron chelators on the testicular function and sperm integrity of patients could not be excluded. In addition, although we demonstrated that sperm quality was impaired in adult male with transfusion-dependent beta-thalassemia, the onset of this functional decline is still unknown. A longitudinal study with a larger sample size might be necessary to determine the onset of the decline in gonadal function and to provide better recommendations for patients with transfusion-dependent beta-thalassemia.

In conclusion, by using MR T2 quantification techniques, we demonstrated that the iron loading representing by the circulating ferritin levels is positively correlated with the testicular MR T2 values, and testicular MR T2 values are significantly lower in patients with transfusion-dependent beta-thalassemia than normal controls. We also found a significantly lower sperm concentration and lower ratio of sperm with normal morphology, and higher SDF ratio in transfusion-dependent beta-thalassemia male patients without hypogonadotropic hypogonadism when compared with those in normal controls. In addition, the SDF ratio was also significantly correlated to serum ferritin levels in our transfusion-dependent beta-thalassemia male patients with spontaneous spermatogenesis. Therefore, this study demonstrated that transfusion-dependent beta-thalassemia males have a high proportion of fertility impairment and iron overload might contribute to disturbed sperm quality and testicular tissue injury. Such findings might explain the high prevalence of impaired fertility in transfusion-dependent beta-thalassemia patients with normal pituitary function and can be generalized to other patients with iron or metal overload syndrome.

\section{ACKNOWLEDGMENTS}

We thank all the patients who participated in this study. We also acknowledge the Taiwan Thalassemia Association for data collection and management.

\section{STATEMENT OF FINANCIAL SUPPORT}

This work was supported by grants from the National Taiwan University Hospital (NTUH 104-S2730, 106-S3399 to H.-H.C.) and Ministry of Science and Technology, Taiwan (MOST 102-2314-B002-132-MY3 to M.-J.C. and 103-2314-B-002-072 to H.-H.C.).

Disclosure: The authors declare no conflict of interest.

\section{REFERENCES}

1. Rund D, Rachmilewitz E. Beta-thalassemia. N Engl J Med 2005;353: $1135-46$.

2. Chern JP, Lin KH, Lu MY, et al. Beta-thalassemia major births after National Screening Program in Taiwan. Pediatr Blood Cancer 2008;50: 58-61.

3. Olivieri NF. The beta-thalassemias. N Engl J Med 1999;341:99-109.

4. Chern JP, Lin KH, Tsai WY, et al. Hypogonadotropic hypogonadism and hematologic phenotype in patients with transfusion-dependent beta-thalassemia. J Pediatr Hematol Oncol 2003;25:880-4.

5. Roth C, Pekrun A, Bartz M, et al. Short stature and failure of pubertal development in thalassaemia major: evidence for hypothalamic neurosecretory dysfunction of growth hormone secretion and defective pituitary gonadotropin secretion. Eur J Pediatr 1997;156:777-83.

6. Bergeron C, Kovacs K. Pituitary siderosis. A histologic, immunocytologic, and ultrastructural study. Am J Pathol 1978;93:295-309.

7. Soliman AT, elZalabany MM, Ragab M, et al. Spontaneous and GnRHprovoked gonadotropin secretion and testosterone response to human chorionic gonadotropin in adolescent boys with thalassaemia major and delayed puberty. J Trop Pediatr 2000;46:79-85. 
8. Bajoria R, Chatterjee R. Current perspectives of fertility and pregnancy in thalassemia. Hemoglobin 2009;33 (Suppl 1): S131-5.

9. De Sanctis V, Vullo C, Katz M, Wonke B, Tanas R, Bagni B. Gonadal function in patients with beta thalassaemia major. J Clin Pathol 1988;41: 133-7.

10. Aessopos A, Karabatsos F, Farmakis D, et al. Pregnancy in patients with well-treated beta-thalassemia: outcome for mothers and newborn infants. Am J Obstet Gynecol 1999;180:360-5.

11. Bajoria R, Chatterjee R. Hypogonadotrophic hypogonadism and diminished gonadal reserve accounts for dysfunctional gametogenesis in thalassaemia patients with iron overload presenting with infertility. Hemoglobin 2011;35:636-42.

12. Olivieri NF, Nathan DG, MacMillan JH, et al. Survival in medically treated patients with homozygous beta-thalassemia. N Engl J Med 1994;331:574-8.

13. Olivieri NF, Brittenham GM. Iron-chelating therapy and the treatment of thalassemia. Blood 1997;89:739-61.

14. Singer ST, Killilea D, Suh JH, et al. Fertility in transfusion-dependent thalassemia men: effects of iron burden on the reproductive axis. Am J Hematol 2015;90:E190-2.

15. De Sanctis V, Soliman AT, Elsedfy H, Soliman NA, Elalaily R. Late-onset male hypogonadism and fertility potential in thalassemia major patients: two emerging issues. Mediterr J Hematol Infect Dis 2015;7:e2015047.

16. Ruder EH, Hartman TJ, Goldman MB. Impact of oxidative stress on female fertility. Curr Opin Obstet Gynecol 2009;21:219-2.

17. Zribi N, Chakroun NF, Elleuch H, et al. Sperm DNA fragmentation and oxidation are independent of malondialdheyde. Reprod Biol Endocrinol 2011;9:47.

18. Cross CE, Halliwell B, Borish ET, et al. Oxygen radicals and human disease. Ann Intern Med 1987;107:526-45.

19. Aitken RJ, Gordon E, Harkiss D, et al. Relative impact of oxidative stress on the functional competence and genomic integrity of human spermatozoa. Biol Reprod 1998;59:1037-46.

20. Jensen CE, Abdel-Gadir A, Cox C, Tuck SM, Wonke B. Sperm concentrations and quality in beta-thalassaemia major. Int J Androl 1996;19:362-4.

21. Safarinejad MR. Evaluation of semen quality, endocrine profile and hypothalamus-pituitary-testis axis in male patients with homozygous beta-thalassemia major. J Urol 2008;179:2327-32.

22. Perera D, Pizzey A, Campbell A, et al. Sperm DNA damage in potentially fertile homozygous beta-thalassaemia patients with iron overload. Hum Reprod 2002;17:1820-5.

23. Nichols-Vinueza DX, White MT, Powell AJ, Banka P, Neufeld EJ. MRI guided iron assessment and oral chelator use improve iron status in thalassemia major patients. Am J Hematol 2014;89:684-8.

24. World Health Organization WHO Laboratory Manual for the Examination and Processing of Human Semen. Geneva: World Health Organization, 2010.

25. Lu JC, Huang YF, Lu NQ. [WHO laboratory manual for the examination and processing of human semen: its applicability to andrology laboratories in China]. Zhonghua Nan Ke Xue 2010;16:867-71.

26. Fernandez JL, Muriel L, Rivero MT, Goyanes V, Vazquez R, Alvarez JG. The sperm chromatin dispersion test: a simple method for the determination of sperm DNA fragmentation. J Androl 2003;24:59-66.

27. Fernandez JL, Muriel L, Goyanes V, et al. Simple determination of human sperm DNA fragmentation with an improved sperm chromatin dispersion test. Fertil Steril 2005;84:833-42.
28. Pavitt HL, Aydinok Y, El-Beshlawy A, et al. The effect of reducing repetition time $\mathrm{TR}$ on the measurement of liver R2 for the purpose of measuring liver iron concentration. Magn Reson Med 2011;65: 1346-51.

29. Cooper TG, Noonan E, von Eckardstein S, et al. World Health Organization reference values for human semen characteristics. Hum Reprod Update 2010;16:231-45.

30. Noetzli LJ, Panigrahy A, Mittelman SD, et al. Pituitary iron and volume predict hypogonadism in transfusional iron overload. Am J Hematol 2012;87:167-71.

31. Wood JC. Diagnosis and management of transfusion iron overload: the role of imaging. Am J Hematol 2007;82:1132-5.

32. Anderson LJ. Assessment of iron overload with $\mathrm{T} 2{ }^{\star}$ magnetic resonance imaging. Prog Cardiovasc Dis 2011;54:287-94.

33. Carpenter JP, He T, Kirk $\mathrm{P}$, et al. On $\mathrm{T} 2^{*}$ magnetic resonance and cardiac iron. Circulation 2011;123:1519-28.

34. St Pierre TG, Clark PR, Chua-anusorn W, et al. Noninvasive measurement and imaging of liver iron concentrations using proton magnetic resonance. Blood 2005;105:855-61.

35. Pathare A, Taher A, Daar S. Deferasirox (Exjade) significantly improves cardiac $\mathrm{T}^{*}$ in heavily iron-overloaded patients with betathalassemia major. Ann Hematol 2010;89:405-9.

36. Wood JC. Magnetic resonance imaging measurement of iron overload. Curr Opin Hematol 2007;14:183-90.

37. Noetzli LJ, Carson SM, Nord AS, Coates TD, Wood JC. Longitudinal analysis of heart and liver iron in thalassemia major. Blood 2008;112: 2973-8.

38. Singer ST, Vichinsky EP, Gildengorin G, van Disseldorp J, Rosen M, Cedars MI. Reproductive capacity in iron overloaded women with thalassemia major. Blood 2011;118:2878-81.

39. Chang HH, Chen MJ, Lu MY, et al. Iron overload is associated with low anti-mullerian hormone in women with transfusion-dependent beta-thalassaemia. BJOG 2011;118:825-31.

40. Mojica-Villegas MA, Izquierdo-Vega JA, Chamorro-Cevallos G, SanchezGutierrez M. Protective effect of resveratrol on biomarkers of oxidative stress induced by iron/ascorbate in mouse spermatozoa. Nutrients 2014;6: 489-503.

41. Agarwal A, Virk G, Ong C, du Plessis SS. Effect of oxidative stress on male reproduction. World J Mens Health 2014;32:1-17.

42. Toumba M, Sergis A, Kanaris C, Skordis N. Endocrine complications in patients with thalassaemia major. Pediatr Endocrinol Rev 2007;5: 642-8.

43. Fernandez-Real JM, Manco M. Effects of iron overload on chronic metabolic diseases. Lancet Diabetes Endocrinol 2014;2:513-26.

44. Goodwin LO, Leeds NB, Guzowski D, Hurley IR, Pergolizzi RG, Benoff S. Identification of structural elements of the testis-specific voltage dependent calcium channel that potentially regulate its biophysical properties. Mol Hum Reprod 1999;5:311-22.

45. Fragale A, Aguanno S, Kemp M, et al. Identification and cellular localisation of voltage-operated calcium channels in immature rat testis. Mol Cell Endocrinol 2000;162:25-33.

46. Wijarnpreecha K, Kumfu S, Chattipakorn SC, Chattipakorn N. Cardiomyopathy associated with iron overload: how does iron enter myocytes and what are the implications for pharmacological therapy? Hemoglobin 2015;39:9-17. 